\title{
Onboard equipment of L- and X-bands synthesized aperture radars for remote sensing of the Earth with higher resolution: requirements and implementation
}

\author{
Mikhail Rovkin ${ }^{1, *}$ \\ ${ }^{1}$ Tomsk State University of Control Systems and Radioelectronics, Research Institute of Electric \\ Communication Systems, 40, Lenin Av., 634050, Tomsk, Russian Federation
}

\begin{abstract}
Requirements to the equipment of dual (L - and X-) band SAR, realizing increase of spatial resolution due to expansion of a spectrum of sounding signals are set. The technical decision of the main components of the onboard equipment and the results of bench tests of the layout of small - sized SAR of L - and X-bands for remote sensing of the Earth prepared for flight tests are described.
\end{abstract}

\section{Introduction}

The expansion of the frequency section of the X-band from 600 to $1200 \mathrm{MHz}$, reserved for aircraft remote sensing radar systems, gave a powerful impetus to the development of SAR operating in this frequency band, since the main trend in the development of such systems is to increase their informativiness, mainly determined by the its spatial resolution [1]. An example of such movement is the further development of the the concept of aviation SAR, developed in recent years in the Research Institute of Precision Instruments (NII TP, Moscow), which is today the main carrier of systems engineering competencies in technoolgy of RSA in Russia, and have them implemented in the experimental multi-band SAR "COMPACT"[2]. The first step of development is implemented in the framework of the university project carried out by Tomsk State University of Control Systems and Radioelectronics (TUSUR) with NII TP as collaborator and Joint-Stock Company "Rrsearch-Production Firm "Mikran" as industrial partner.

The main goal of the project is phased creation of two prototype of dual-band remote sensing radar and their subsequent flight tests. A significant (approximately twice up to $1000 \mathrm{MHz}$ at X- band and up to $400 \mathrm{MHz}$ and at L-band) expansion of the spectrum of the sounding signal forces us to re-create all the basic elements of the SAR in both frequency bands: more broadband antenna systems, and microwave radio paths, including output powerful amplifiers of transmitter as well as digital paths for the formation of the sounding signal and processing of the radar response. In addition, to increase the spatial resolution in the direction of synthesis it was necessary to double the width of the main lobe of the SAR antenna pattern in azimuth. To compensate for the inevitable decrease in the energy

* Corresponding author: mikhail.rovkin@tusur.ru 
potential of the radar due to the increase in the noise coefficient of the receiving path by expanding its bandwidth and reducing the antenna gain by expanding the main lobe of its anenna pattern, it was necessary to increase the radiation power.

Along with the increase in the extension of the spectrum of the sounding signal of the SAR and its energy potential (characterized by typical pulse power of $250 \mathrm{~W}$ at duty cycle of 20 and the duration of $60 \mu \mathrm{s}$ ) the task of reducing the size and weight of the onboard equipment were solved, the aggregate of which is a controversial task. The main technical solutions for base units of SAR and their technical characteristics achieved during the development of first model during bench tests are described in the paper.

\section{Antenna system}

Antennas for two frequency bands are separate. For the first flight tests, two L-band antennas and one $\mathrm{X}$ - band antenna were manufactured and tested.

\subsection{L-band antennas}

L-band antennas both had linear but different - vertical and horizontal - polarization. They were built according as 6 -element $2 \times 3$ arrays. Design features are clear from the photos of the antennas, presented at Fig.1.
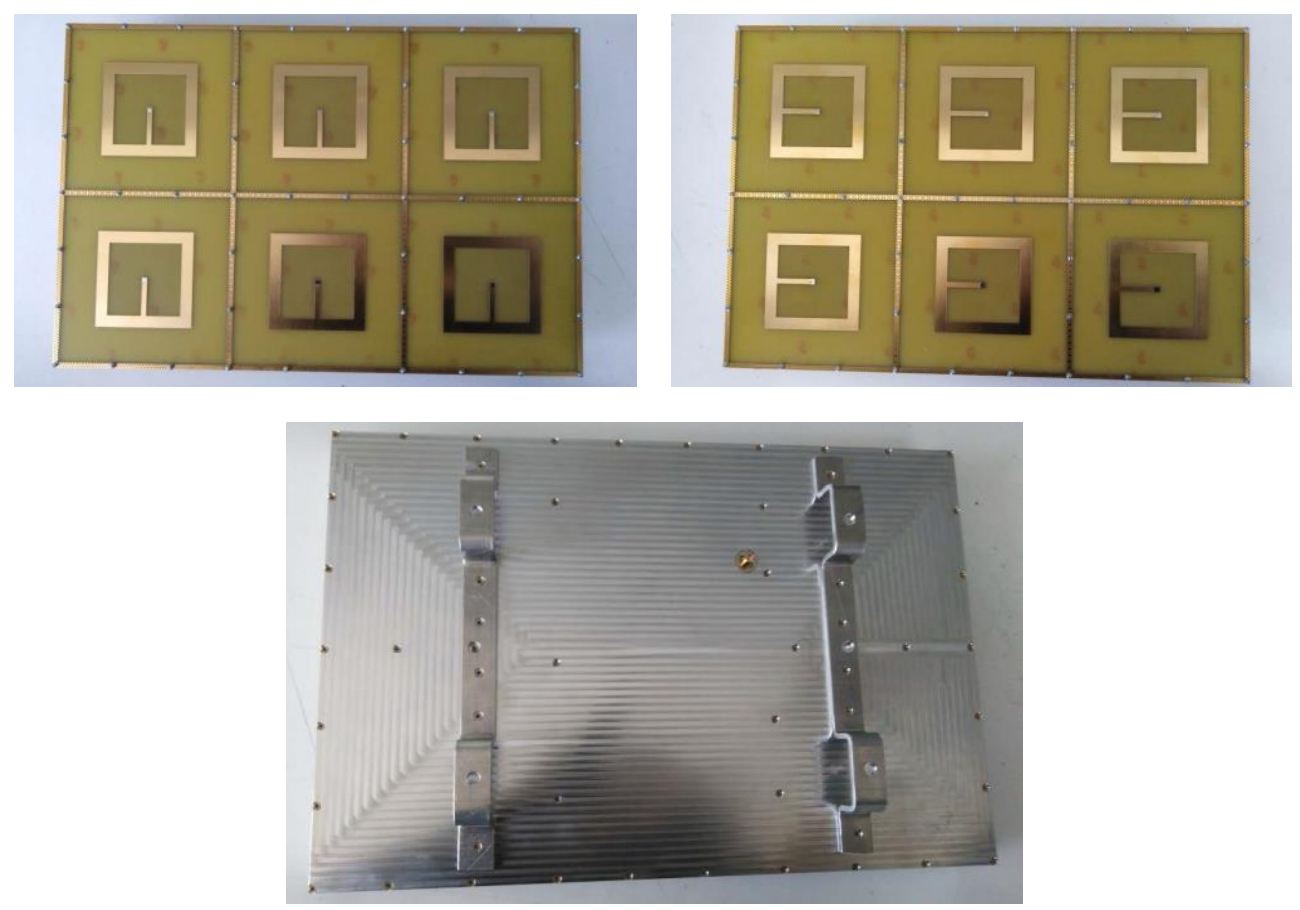

Fig. 1. L-band antenna design: vertical polarization antenna, aperture view (top left), horizontal polarization antenna, aperture view (top right), back side with fastening elements (bottom).

Main element type of both array was micro strip frame as providing a wide bandwidth ( $40 \%$ of central carrying frequency). The results of measurements of the antenna characteristics (including those carried out in the anechoic chamber) are summarized in the Table 1. The main developer of this antennas was a certified engineer Maxim A. Plotnikov (TSUCSR). 
Table 1. Measured values of obtained technical characteristics of L-band antennas.

\begin{tabular}{|c|c|c|}
\hline \multirow{2}{*}{ Parameter } & \multicolumn{2}{|c|}{ Obtained value } \\
\cline { 2 - 3 } & V-polarization antenna & H-polarization antenna \\
\hline Bandwidth, MHz & $<1.57$ & $<10-1510$ \\
\hline VSWR & $<4-32.7(37.2-34.8)$ & $34,9-49.6(25.8-33.2)$ \\
\hline Main lobe width at E-plane (H-plane), ${ }^{\circ}$ & $<-11.7(<-18)$ & $<-11(<-11,9)$ \\
\hline Side lobe width at E-plane (H-plane), dB & $<-18.2$ & $<-18.2$ \\
\hline Cross-polarization level, dB & $>11.9$ & $>11.4$ \\
\hline Gain, dB & $400 \times 270 \times 70$ & $400 \times 270 \times 70$ \\
\hline Size, $\mathrm{mm}$ & 3 & 3 \\
\hline Weight, $\mathrm{kg}$ & & \multicolumn{2}{|c|}{1010} \\
\hline
\end{tabular}

\subsection{X-band antenna}

Developed X-band mirror antenna having horizontal linear polarization had a mass of $650 \mathrm{~g}$ (Fig.2). The results of measurements of the antenna characteristics are summarized in the Table 2. The main developer of this antennas was a certified engineer Maxim A. Plotnikov (TSUCSR).
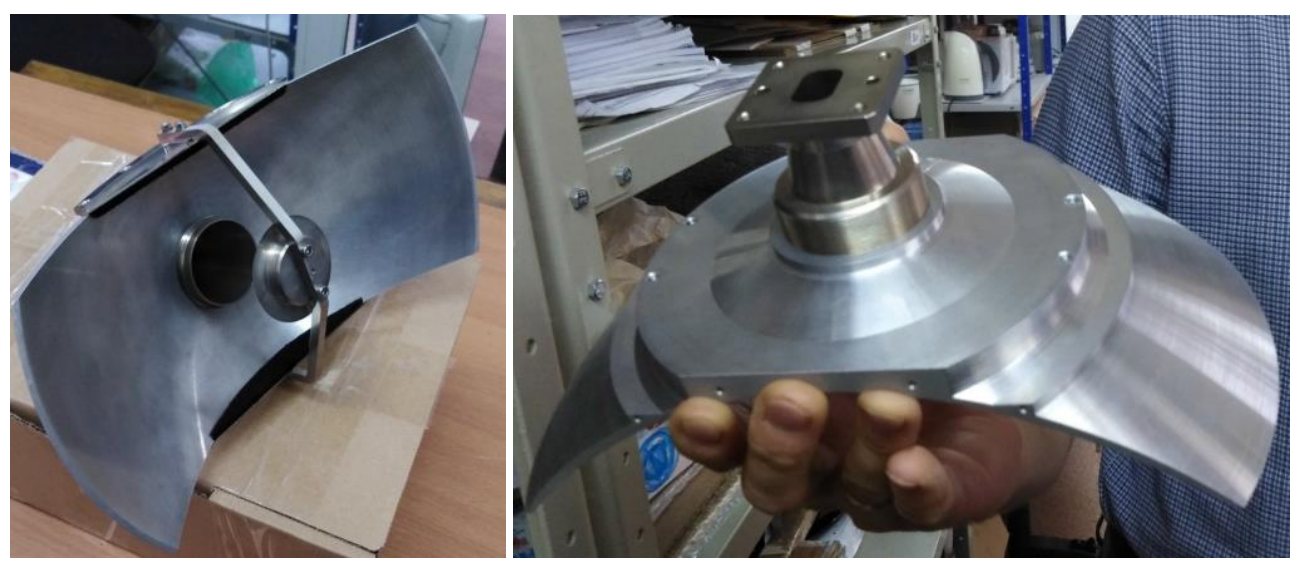

Fig. 2. X-band antenna design: aperture view (left), back side view (bottom).

Table 2. Measured values of obtained technical characteristics of $\mathrm{X}$ - band antennas (polarisation $\mathrm{H}$ ).

\begin{tabular}{|c|c|}
\hline Parameter & Value \\
\hline Bandwidth, MHz & $9300-10300$ \\
\hline VSWR & $<1.35$ \\
\hline Main lobe width at E-plane (H-plane), dB & $7.3-8(14.4-15.3)$ \\
\hline Side lobe width at E-plane (H-plane), dB & $<-15.8(<-13.4)$ \\
\hline Cross-polarization level, dB & $<-22.8$ \\
\hline Gain, dB & $>20$ \\
\hline Size, mm & $300 \times 200 \times 150$ \\
\hline Weight, g & 650 \\
\hline
\end{tabular}




\section{Power amplifiers}

Building of the power output amplifier of both frequency bands is a complex technical task. These units are very critical and determine the mass and size of the transceivers in both frequency ranges of the SAR. Achievement of ordered size and weight for given linearity characteristics was possible to due to the use of modern power $\mathrm{GaN}$ transistors.

The X-band linear power amplifier has a block-modular design, its design was described in detail in [3]. It provides a pulse output power of more than 300 watts in the frequency range of $1 \mathrm{GHz}$ at $3 \mathrm{~dB}$ compression, which confirms the frequency dependence of its output power, shown at Figure 3 below. The Fig. 3 also shows the appearance of this powerful amplifier. Main developer of this amplifier was a certified engineer Anton V. Shabash (TSUCSR).
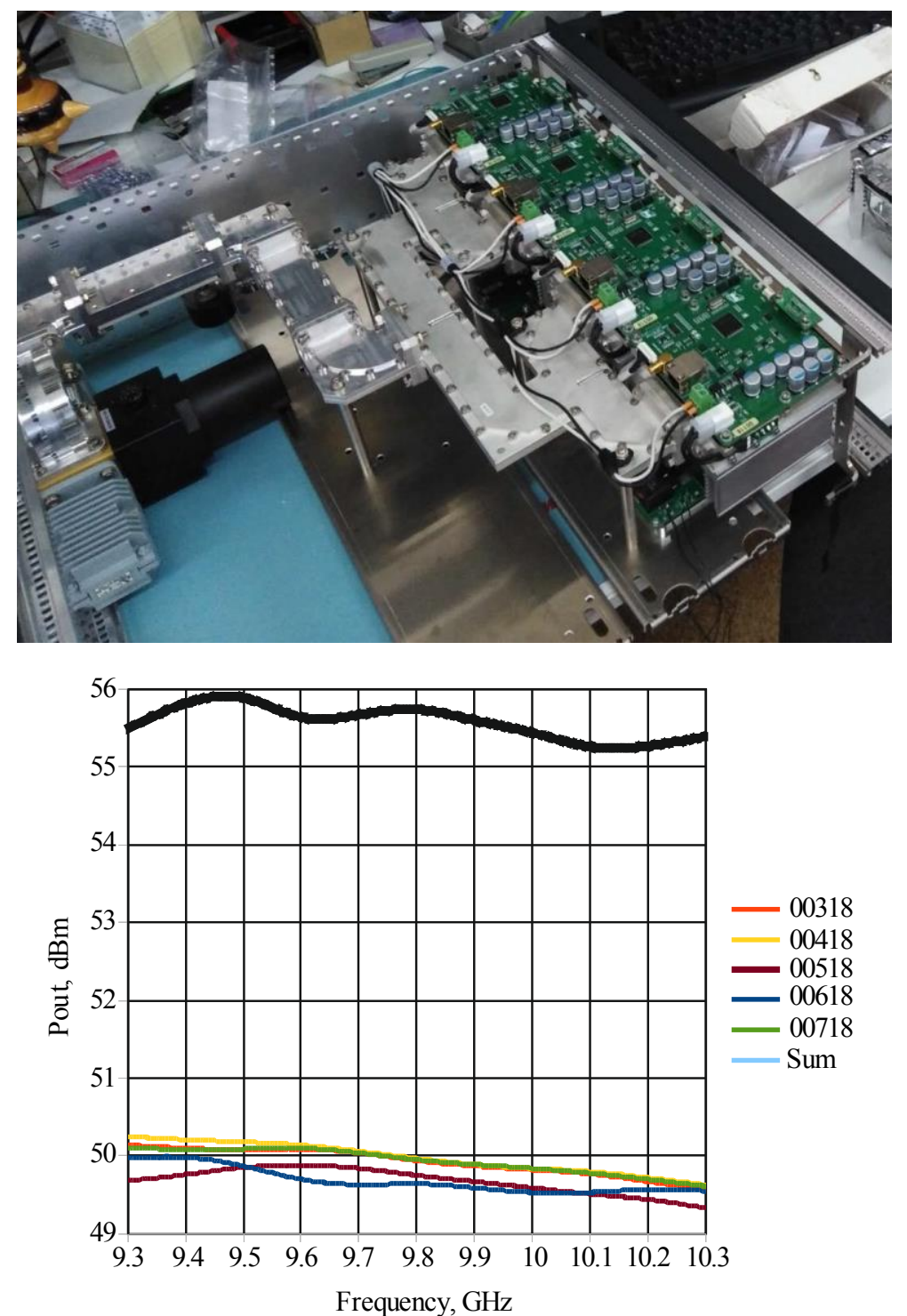

Fig. 2. X-band power amplifier with feeder elements mounted in X-band transiever (top) and frequency dependecies of output power of its four submodules and sum power and bandwidth. 
An output stage of power L-band amplifier was made as single class-A scheme based on Quorvo GaN transistor and provides a pulse output power of more than $270 \mathrm{~W}$ in the frequency range of $400 \mathrm{MHz}$ with $3 \mathrm{~dB}$ compression. The amplifier was developed by group of certified engineer headed by Mikhail V. Dugin (TSUCSR).
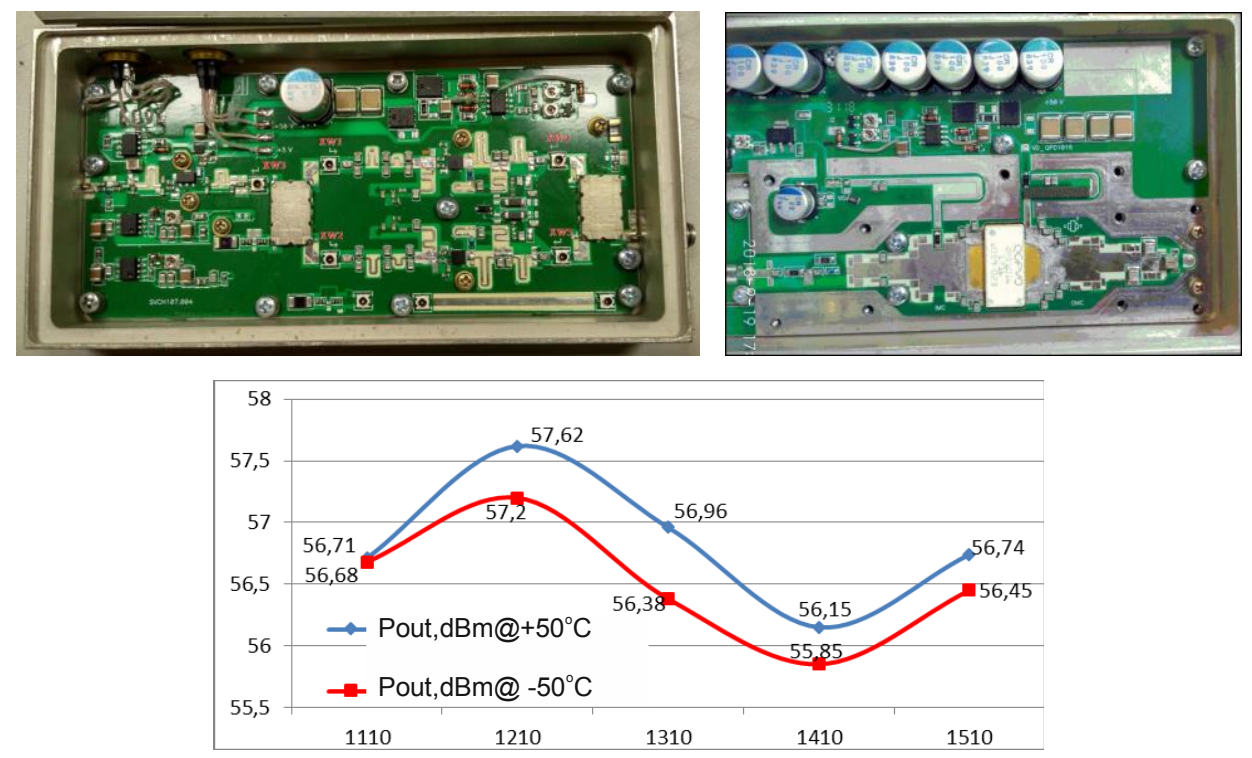

Fig. 3. L-band power amplifier view : input stages (left top), output stage (right top) and frequency characteristics of output power at standard termocycle (bottom).

\section{Receiving-transmitting convertors and general layout of transceivers}

An unified scheme of frequency transformation with direct quadrature modulation in the formation of the sounding signal and demodulation in the reception of radar response was implemented In the transceivers of both bands. Features of construction of transceivers, and also arrangement of transceivers was in detail described earlier in work [4]. Obtained in both ranges of relatively high noise figure $(5 \mathrm{~dB})$ due to the losses made to the input protective devices of radar receivers operating at a high level of leakage of radiated signal that is caused by substantially imperfect matching of the antennas in the extended frequency range. An important feature of the receiving paths is the possibility of operational software adjustment of their transmission coefficients within $35 . .65 \mathrm{~dB}$ while maintaining a constant noise figure, that is important for operating (remote sensing) at wide range of radar reflectivity of sounded real Earth covers.

The transceivers were packaged in standard 19-inch 2U (L-band) and 3U (X-band) packages, respectively. Fig 4 shows appearance of L-band transmitter.
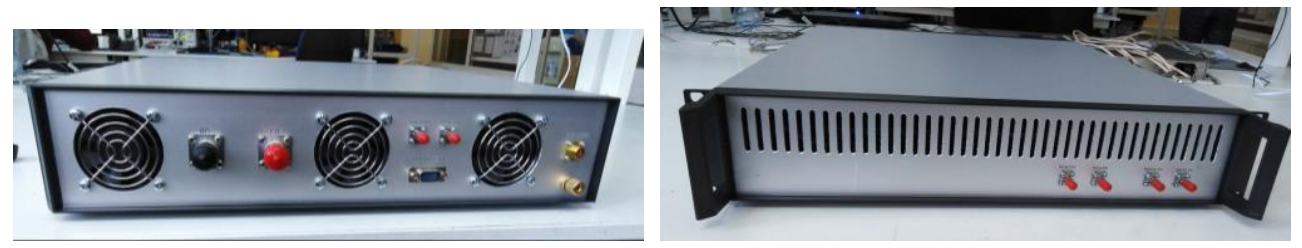

Fig. 4. Appearance of L-band transceiver. 
The final design of converters is performed Anton Yu. Ryzhov (TSUCSR) in support of chief specialist Andrey N. Gusev (Mikran). The general design of the transceivers was performed by leading engineer Dmitry N. Mysik (TSUCSR).

Obtained Weight of MW transceivers of first prototype was $10 \mathrm{~kg}$ (L-band) and $14 \mathrm{~kg}$ (X-band), respectively, that at all corresponds to technical specification.

\section{Wide spectrum digital signal formation and processing in the new SAR}

As was signed above, RF path of SAR built on as a single direct conversion [4] frequency quadrature modulator in the transmit path and a demodulator in the receive path, as providing the maximum possible frequency band of the signal radiation and reception of SAR when processing them. At the same time, complex microwave signals of radiation and radar response are fully characterized by pairs of quadrature components formed by twochannel DAC and ADC, respectively. Digital radio paths of the new SAR, concept of which was described in report [5], structurally and constructively combined with on-board control computers (Fig.5), implemented on the base of high speed DSP units developed by JSC "Instrumental Systems" (Moscow), specially modernized for our project (Fig 6), that allows to form and coherently receive radio signals with a spectrum width of up to $1 \mathrm{GHz}$.
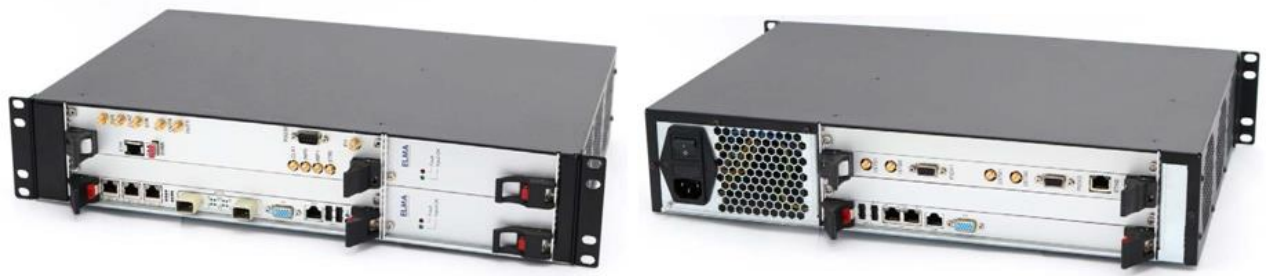

Fig. 5. Appearance on-board control computer.

Sampling frequency at AD and DA conversion was $1400 \mathrm{MHz}$. Front-end was realized on a pair of Kintex7 heavy FPGA. First FPGA operates at Tx (with DAC), second FPGA at $\mathrm{Rx}$ (with ADC)). Inputloutput digital streams describing samples of quadrature components of RF radar signal flows via PCI-Ex lanes through PCI-Ex hub to digital convolver was realized on a pair of TI TMS6768 eight-core DSP. All units (FPGA and DSP) was equipped with 256MB RAMs.
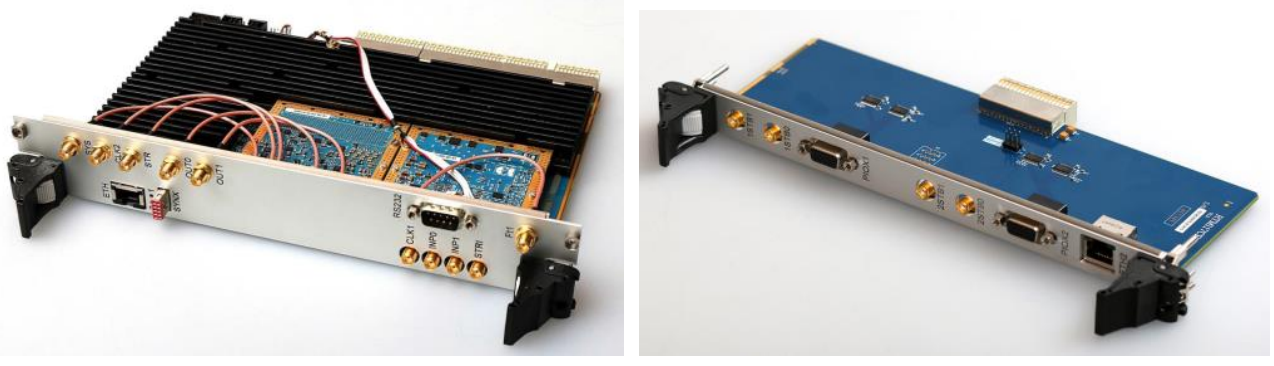

Fig. 6. Appearance of modernized DSP module (left) and new rear module (right) realized for new SAR.

Such scheme of digital signal processing allows reduce the volume of stored output data due to the fact that the convolver handles a limited interval at distance with duration close to sounding pulse and duty cycle of sounding signal is at least 20 . Therefore, the average speed of the digital output stream is reduced, which allows you to save it using typical storage devices Output digital signals is registered on solid disk drive. 


\section{On-board equipment on the first flight test}

Previously tested on-board equipment set was mouned (Fig.7) at the end of June 2019, on the board of flying laboratory IL-18D № 75713 (Fig.8). First flight test, cover main part (but not wholeset) of conditions modes was done. Detailed discussing results of that should be done later in next reports and articles, but we can show first high-resolution images (Fig.8). Flying test was organized by NII TP with "MIR" aviation company.
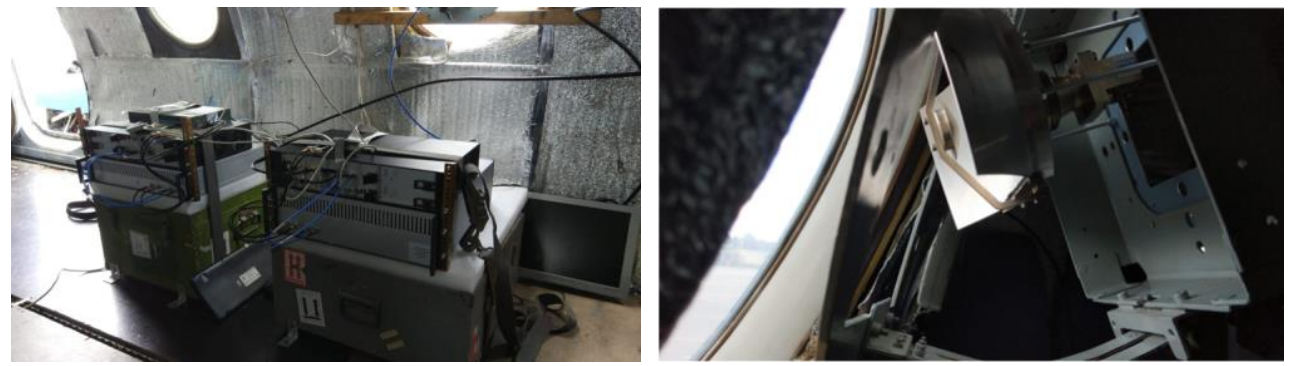

Fig. 7. Transmitters with on-board computers of both bands left) and X-band antenna (right) on the board of flying laboratory.

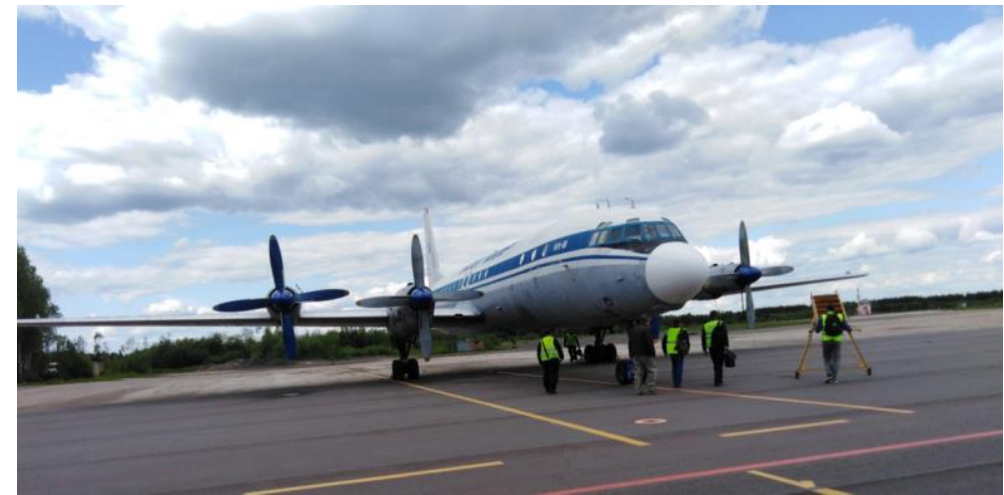

Fig. 8. Flying laboratory IL-18D № 75713 before first test flight. Morning of 28 June, 2019.
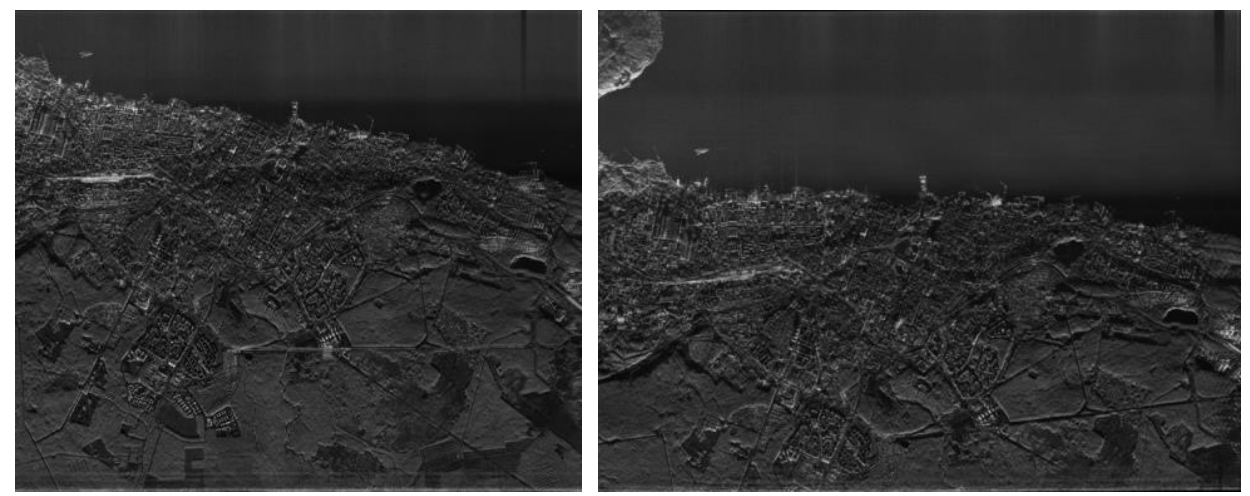

Fig. 9. Examples of radar images processed from focused radio holograms obtained from results of radar sounding at L- and X-band in 1 July, 2019, near Petrozavodsk from the board of flying laboratory IL-18D № 75713 with new on-board eqipments.

Tests were carried out by R.V. Ermakov, M.Yu. Dostovalov (both NII TP), M.S.Samuleev and M.E. Rovkin (both TSUCSR). 


\section{Conclusion}

First half of long roadmap targeted on building of new high-resolution and SAR was done. Some results was obtained and conclusion can be done.

1. Developed equipment was tested in real condition and environment. Main units shows its operability.

2. Most previously evaluated technical characteristics was confirmed experimentally in wide (but not a whole) set of flight modes (heights of flights and soundings was not greater than $3600 \mathrm{~m}$ ). Main results is that spatial resolution in tested modes is close to calculated value.

3. Some features of equipmets functioning was taked into account at design of the second prototype of new dual-band SAR.

4. Concept of SAR was developed is feasible and potentially scalable focusing on visible trends of today digital and MW technology.

Author thanks spetialists of NII TP, especially T.G. Moussiniants and Roman V. Ermakov for useful discussions.

The research and publication were supported by the Ministry of Education and Science of the Russian Federation under the agreement of 14.577.21.0279 of 26.09.2017, identifier RFMEFI57717X0279.

Experimental studies were performed using the equipment of the Center for collective use "Impulse".

\section{References}

1. S.L. Vnotchenko, T.G. Moussiniants, R.V. Ermakov, M.E. Rovkin. XIV Int. Sci.-Tech. Conf. on Act. Prob. of Electr. Instr. Eng. (APEIE) 2018. Modern Tendencies in the Development of Airborne Synthesized Aperture Radars for Remote Sensing of the Earth, 439, DOI: 10.1109/APEIE.2018.8545651 (2018)

2. S.L. Vnotchenko, M.Yu. Dostovalov, A.V. Dyakov at al. Proc. of XXVIII Rus. Symp. "Rad. Inv. of Nat. Env." St.P.,2013, Aviation four-band radar complex "COMPACT" features, results and development prospects. 1, 34 (in Russian) (2013)

3. A.V. Shabash, A.V. Komendantenko, M.E. Rovkin. 2019 U. Symp. on B. Eng., Re. and Inf. Tech. (USBEREIT), Yekaterinburg, Russia 25-26 Apr. 2019, Modular Power Amplifier for Transmitter of X-band Aircraft High Resolution SAR for Earth Remote Sensing. DOI: 10.1109/USBEREIT.2019.8736590 (2019)

4. A.N. Gusev, A.V. Ryzhov, M.S. Samuleev, M.E. Rovkin. Proc. of 28th Int Crim. Conf. MW and Telecom. Tech. (CriMiCo'2018) Sevastopol, 9-15 Sep. 2018- Moscow, Minsk, Sevastopol., Transmit-receive path with extended frequency bandwidth for high-resolution aviation SAR. C, 1749 (in Russian) (2018)

5. I.V. Dyakov, R.V. Ermakov Proc. of 28th Int Crim. Conf. MW and Telecom. Tech. (CriMiCo'2018) Sevastopol, 9-15 Sep. 2018 Moscow, Minsk, Sevastopol., Digital path of formation and processing of the signal for new generation SAR, C, 1755 (in Russian) (2018) 